\title{
A study of the effect of the lung shape on the lung absorbed dose in six standard photon and neutron exposure geometries
}

\author{
A. Ebrahimi Khankook, H. Miri Hakimabad ${ }^{\star}$ and L. Rafat Motavalli \\ Physics Department, College of Sciences, Ferdowsi University of Mashhad, 91775-1436 Mashhad, Iran.
}

Received 19 June 2014 - Accepted 31 October 2014

\begin{abstract}
According to the published results of radiation dosimetry studies, there are significant discrepancies in the organ absorbed doses of existing adult male phantoms. As stated, differences in the organ absorbed doses may be associated with the variations in the organs' volumes, shapes and positions in the body frame. Therefore, this paper focuses on the effect of the lung shape on the lung absorbed dose by creating a series of voxel phantoms, in which the lung shape follows a statistical distribution. These phantoms were exposed to mono-energetic photons and neutrons in six standard irradiation geometries. The results show that when the phantom is irradiated by the low-energy photons, the effects of the lung shape on the lung absorbed dose are considerable (with an uncertainty of more than $100 \%$ ). For the other irradiation conditions, the variation in the lung shape causes an uncertainty of less than $10 \%$ in the dose delivered to the lung.
\end{abstract}

Keywords: dosimetry / statistical phantom / lung shape / MCNP5

\section{Introduction}

Estimation of the radiation dose delivered to the human body from external sources requires a certain amount of anatomical data about the exposed individual. According to the Task Group on Reference Man of the International Commission on Radiological Protection (ICRP), the mass, dimensions, and elemental composition of the organs and tissues are essential parameters for dosimetric calculations (ICRP, 1975). In 2002, the task group introduced the anatomical and physiological data of a well-defined reference individual at the 50th height/weight percentile of the Caucasian population as an instrument to ensure the compliance of actual doses with the legally established dose limits. Hence, dose values assessed by ICRP Reference Man are representative of a large population and do not provide a precise dose value for any individual persons (ICRP, 2002).

In recent years, several reference phantoms have been developed based on the ICRP published sets of anatomical data (Kramer et al., 1982, 2003, 2004, 2006; Zankl and Wittmann, 2001; Ferrari and Gualdrini, 2005; ICRP, 2009; Zhang et al., 2009b; Cassola et al., 2010). In addition, researchers developed some Asian reference phantoms based on the Asian Standard Man data (Saito et al., 2001; Lee et al. 2004, 2006; Sato et al., 2007; Zhang et al., 2007; Li et al., 2009). Although the discrepancies between the organ masses and volumes of different Caucasian reference phantoms are fairly small, their

\footnotetext{
* mirihakim@ferdowsi.um.ac.ir
}

organ doses in the photon fields differ significantly in some situations (Zankl et al., 2001; Ferrari et al., 2005; Zhang et al., 2009b; Kramer et al., 2010). Such differences have also been observed in the resultant organ doses calculated using various Asian reference phantoms (Saito et al., 2009; Zhang et al., 2009a).

Comparisons between existing adult male phantoms indicated that the differences in the organ doses, assessed for external exposures, might be affected by several factors (Saito et al., 2001):

- total body height/weight;

- initial anatomy which the phantom is constructed based upon, including the sizes, shapes, and positions of organs and tissues;

- the transport calculation method.

The first factor is a major parameter, which affects organ doses in external radiation exposure. Considering the results of Karimi-Shahri et al., organ absorbed doses strongly depend on the whole-body size (Karimi-Shahri et al., 2013). However, all reference phantoms of a particular race (Caucasian or Asian) have similar whole-body sizes. So, this factor cannot be the major reason for the differences mentioned. On the other hand, according to ICRP Publication 116, the data sets generated from different Monte Carlo codes (EGSnrc, MCNPX 2.6.0 and GEANT4 for photons and PHIT, Fluka and MCNPX 2.5.0 for neutrons) revealed that the variations among the results were relatively small. In the worst situation, the transport calculation method may result in up to $10 \%$ differences in organ absorbed doses (ICRP, 2010). 
According to the above considerations, the anatomical differences of reference phantoms lead to major variations in organ absorbed doses (Zhang et al., 2009b; Kramer et al., 2010). It is worth mentioning that the ICRP reference values are intended to depict the average organ volumes and masses, without specifying the organ shapes and positions (ICRP, 2002). Thus, other phantoms that match the reference parameters are also representative of the 50th percentile of the adult population. In reality, however, the shapes and locations of the organs differ slightly; each phantom will exhibit different dosimetric features.

Recently, with the enormous increase in the processing performance of computers, accurate calculations have become very demanding in the field of ionizing radiation dosimetry. However, there are a large number of similar phantoms with slight differences in the anatomical details, which can affect the organ dose level. Thus, to provide a dose data library, it is necessary to report the organ absorbed doses and their uncertainties due to the anatomical features. The method described in this paper is implemented based on the proposal of Professor Xu to quantify the uncertainty of the organ absorbed dose by constructing a series of deformable phantoms with anatomical parameters that follow statistical distributions ( $\mathrm{Xu}$ and $\mathrm{Liu}$, 2011).

The current manuscript extends the statistical models of lung phantoms by considering the lung shape as a statistical parameter, and investigates the effects of the lung shape on the lung absorbed dose in different irradiation geometries. To maintain the generality of this approach, mono-energetic photons and neutrons in six standard irradiation geometries were considered. Importing 70 lung models into the simplified adult male ICRP phantom, the averaged lung absorbed dose per fluence (LADF) and its standard deviation were calculated for a total of 258 irradiation conditions ( 24 mono-energetic photon sources $\times 6$ standard geometries +19 mono-energetic neutron sources $\times 6$ standard geometries). Previously, the effects of the lung mass variations on the lung absorbed dose were studied by Ebrahimi Khankook et al. (2014).

\section{Materials and methods}

\subsection{Creation of statistical lung models}

In order to provide statistical phantoms by considering the lung shape as a statistical parameter, 70 lung models were extracted from chest computed tomography (CT) images collected from the Imam Hossein Hospital of Tehran, Imam Reza Hospital of Mashhad and Parsian Medical Imaging Center of Mashhad databases. The chest CTs belong to patients with normal lungs, so that diseases did not change the size or shape of these lungs. These lungs were segmented manually by the segmentation team using 3D-DOCTOR ${ }^{\mathrm{TM}}$ software (Able Software Corp., Lexington, MA).

Because of the high density of the bone, the rib cage plays a significant role in the shielding of the lung tissue. On the other hand, the shapes of the lung and rib cage are strongly related to each other. Thus, in addition to the lung tissue, the segmentation of the rib cage is also required.
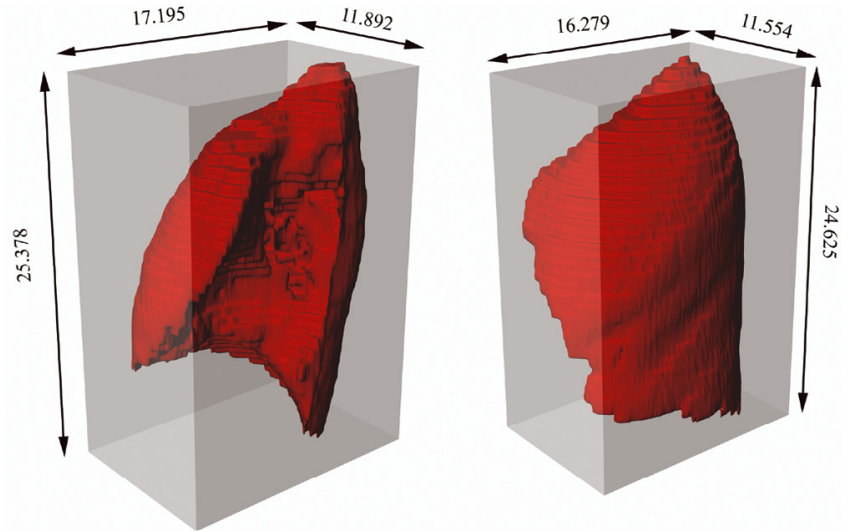

Fig. 1. Dimensions of boundary boxes of the right (a) and left (b) lobes of the normal lung (ICRPlung).

To minimize the effects of lung masses on LADF measurements, extracted models were fitted to the desired lung reference volume using Rhinoceros ${ }^{\mathrm{TM}}$ software (McNeel North America, Seattle,WA), so that the size of the right lobe was about $18 \%$ greater than that of the left lobe. Relative errors between the resultant lung mesh volumes and ICRP recommended values for the left and right lobes were below $1 \%$. After changing the lung volume using the scale option of the Rhinoceros software, the rib cage was resized to match the new lung boundary.

To ensure that the lung models were selected statistically, distributions of maximum height, width and depth were investigated by calculating the dimensions of the boundary box of each lobe (see Fig. 1). In the resultant lung models, the maximum height, width and depth of each lung's lobe (left or right) follows a normal distribution. Figure 2 shows the distributions of the height, width and depth of the left lung lobe. Surface rendering of 16 cases is also given in Figure 3.

\subsection{Extending the lung models to the whole-body frame}

The shape of the body, the thickness of the muscle around the lung, the position of the arms, and the location of the lung in the body can affect the lung absorbed dose significantly. In order to provide similar conditions for the irradiation, the Adult Male ICRP reference phantom (AM) was considered as a template phantom.

Because of the difficulties in deforming an organ, adjusting its volume and also avoiding the overlaps due to using a voxel phantom, a mesh representation of the AM phantom was needed. Creating a mesh model was performed by providing a set of digital images in the DICOM (digital imaging and communications in medicine) format using the algorithm implemented in MATLAB ${ }^{\circledR}$ 7.4. Each image consists of a matrix of pixels, whose color depends on the identification number of corresponding voxels in the voxel matrix. To assign organ boundary information to each of the images and to export the organ mesh model, the segmentation process was performed by the 3D-DOCTOR software. 

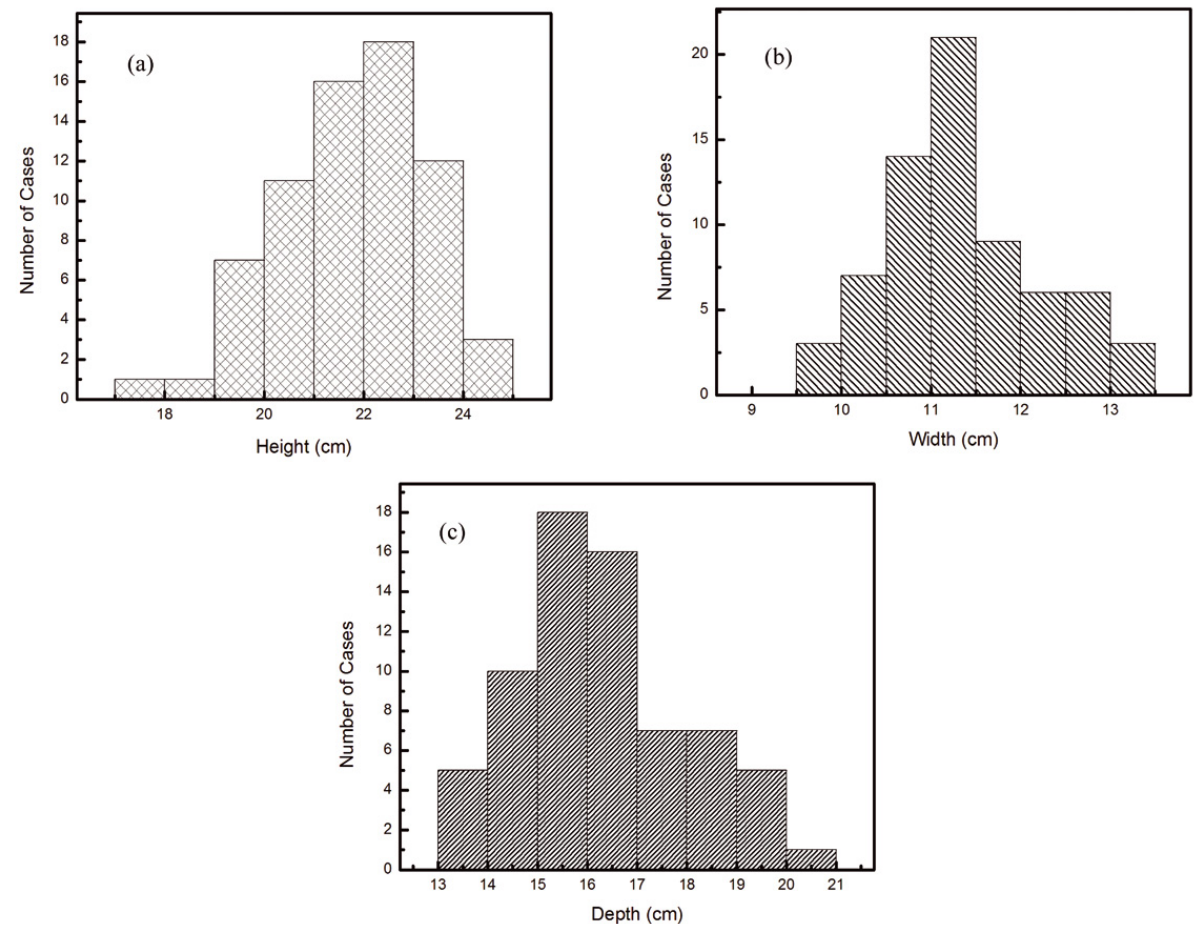

Fig. 2. The distributions of the height (a), width (b), and depth (c) of the left lungs lobes.
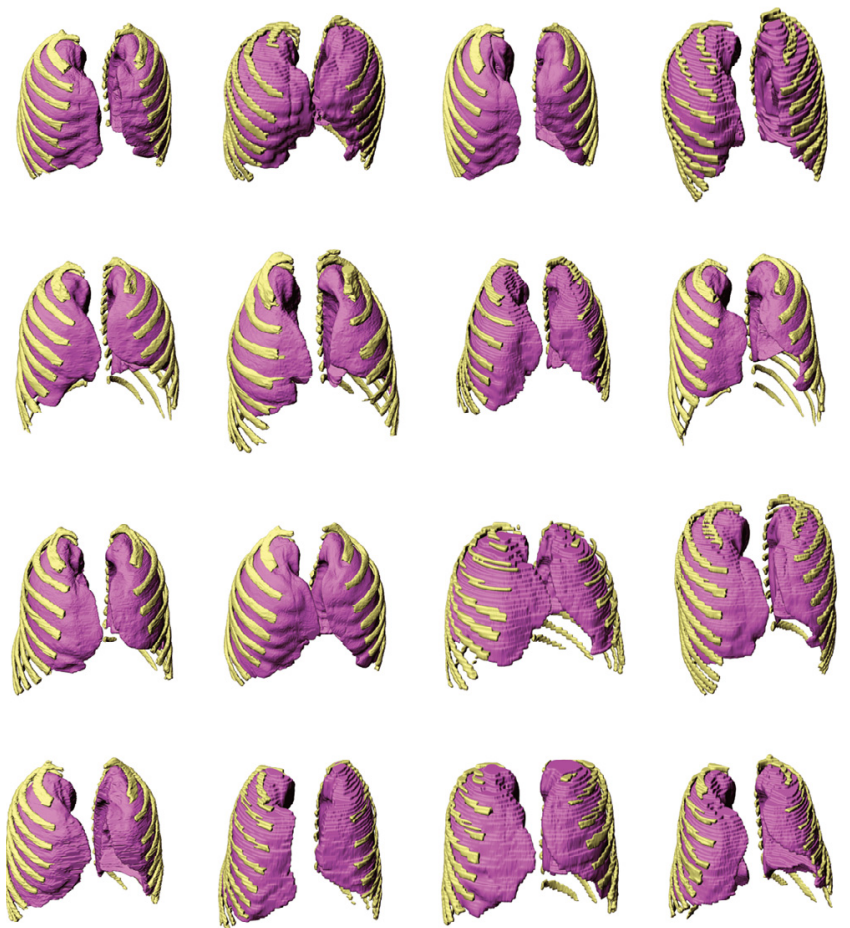

Fig. 3. Surface rendering of 16 cases of the segmented lungs and rib cages.

Before integrating new models of the lung and rib cage into the whole-body frame, some major modifications were carried out carefully. To avoid the possible overlaps between the new lung and rib cage and its adjacent organs, during the process of replacing and adjusting the location, internal organs and tissues of the AM phantom were replaced by soft tissue (with the averaged density of $1.05 \mathrm{~g} / \mathrm{cm}^{3}$ ) except for bone. This is due to the fact that bone has high density and can play a significant role in the shielding of the lung tissue. The potential overlaps of the inserted models, including the lung and rib cage, and adjacent bone groups such as the spine (cervical, thoracic and lumbar), scapulae and clavicles, were carefully resolved by minor displacement of these organs. Finally, the lung and rib cage of the AM phantom were removed and replaced by new lung and rib cage models. Because of the effects of the organ location on the organ absorbed dose, for comparability of the results, the centers of mass of the left and right lobes were fixed at $(34.86 \mathrm{~cm}, 17.498 \mathrm{~cm}$ and $137.2 \mathrm{~cm})$ and $(20.92 \mathrm{~cm}$, $16.488 \mathrm{~cm}$ and $137.1 \mathrm{~cm})$, respectively. Figure 4 shows different views of one of the statistical phantoms constructed by this method. The positions of the bone groups, which have been highlighted in gold, were slightly modified.

For the purpose of dosimetry calculation using the MCNP5 Monte Carlo code, resultant phantoms were subsequently converted to the voxel format by a voxelization algorithm implemented in Fortran 90. According to the resolution of the AM phantom, the voxel resolution of final models was $2.137 \times$ $2.137 \times 8 \mathrm{~mm}^{3}$.

\subsection{Monte Carlo simulation}

In the present paper, dosimetry calculations were performed using MCNP5 Monte Carlo code. Six standard exposure geometries, anterior-posterior (AP), posterior-anterior (PA), left lateral (LLAT), right lateral (RLAT), rotational (ROT) and isotropic (ISO), were considered for uniform photon and neutron fields. 24 mono-energetic photon sources 

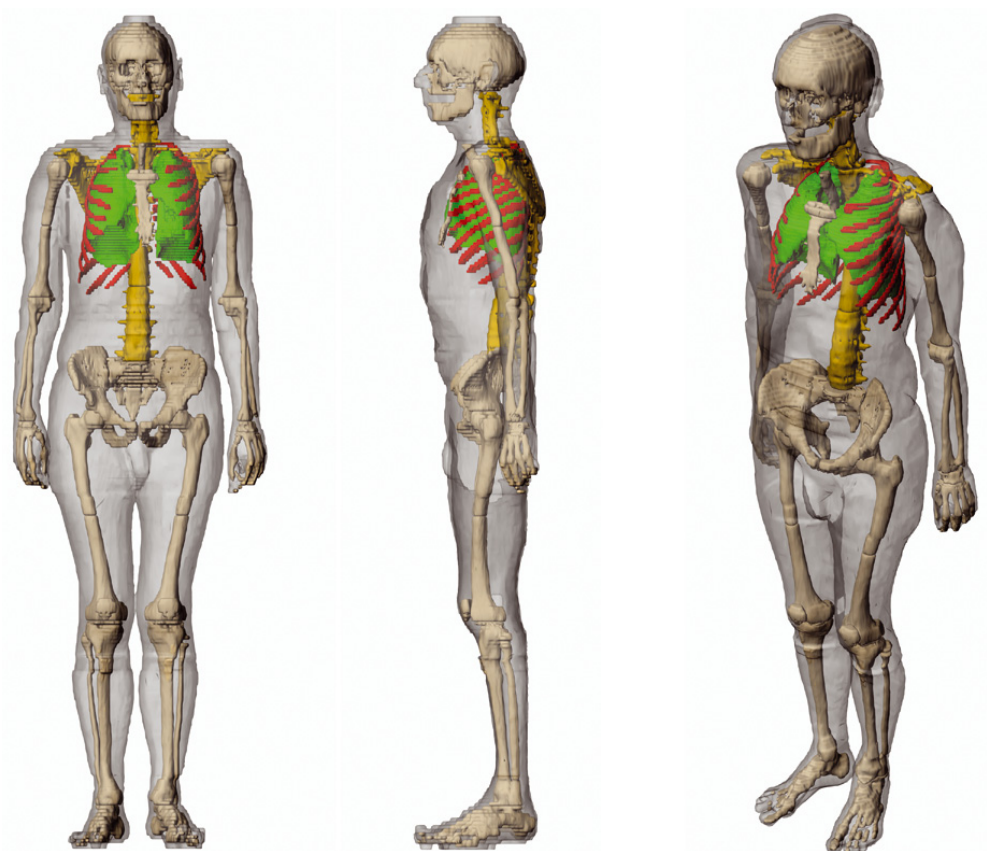

Fig. 4. One of the whole body phantoms used in this paper. The positions of bone groups, which have been highlighted in gold, were slightly modified. The whole-body frame is filled by the soft tissue, ignoring the lung, rib cage, and skeleton.

covering a range of $15 \mathrm{keV}$ to $10 \mathrm{MeV}$ and 19 mono-energetic neutron sources including a range of $10^{-8} \mathrm{MeV}$ to $20 \mathrm{MeV}$ were considered for the simulation.

To estimate the organ absorbed dose, the energy deposition tally of track length estimation, $* \mathrm{~F} 8$, with the unit of energy deposited per source particle was applied. The result of the *F8 tally was divided by the mass of the cell and the absorbed dose was provided. Since the kerma approximation is valid for low-energy photon sources, only deposited energy of photons was considered. Above $500 \mathrm{keV}$, the secondary charged particle equilibrium is not fully established in the lung and especially rib cage, and thus electron transport was explicitly performed. Therefore, secondary electrons were also tracked for energies above $500 \mathrm{keV}$. However, at neutron energies below $20 \mathrm{MeV}$, compared with the organ dimensions, the ranges of the secondary charged particles are very short, so that the absorbed dose was computed by means of kerma approximation. Therefore, the F6 tally was used to calculate the organ dose due to neutrons and photon-induced neutrons.

It is to be noted that the associated relative errors scored with each energy deposition in the lung were below $0.5 \%$, except for the photons with energy of $15 \mathrm{keV}$, which had uncertainties of around $3 \%$. The number of source photons transported in the Monte Carlo simulations was $2 \times 10^{9}$ for $15 \mathrm{keV}$, $1 \times 10^{8}$ for $20-500 \mathrm{keV}$, and $1 \times 10^{7}$ for the higher energies. The computation time for different energies varied from 100 to 5000 minutes. In addition, for neutron sources, $1 \times 10^{8}$ particle histories were tracked and the average execution time for each case was around 100 minutes. The total execution time for all simulations reported in this work was 52200 core-hours. A personal computer equipped with a $3.06 \mathrm{GHz}$ Intel core i7 processor and 6 GB RAM, operated by Microsoft Windows 7, was used in computations.

\subsection{Statistical parameters}

The uncertainties of the LADF due to the lung shape variation can be described by the standard deviation (SD) of the LADF. SD is a very profitable parameter for determining the amount of the LADF error in different radiation types, energies and geometries:

$$
S D=\sqrt{\frac{\sum\left(x_{i}-x^{\prime}\right)^{2}}{N}}
$$

The coefficient of variation $(\mathrm{CV})$ is also used to compare the deviation of LADF data from the mean value for the various forms of the radiation exposure.

$$
C V=\frac{S D}{x^{\prime}}
$$

\section{Results}

\subsection{Mono-energetic photon sources}

Table 1 presents the average LADFs and their SDs for mono-energetic external photon exposure in standard geometries. Regardless of the irradiation geometry, the statistical fluctuation of the LADF for low-energy photons is considerable. However, by increasing energy above $80 \mathrm{keV}$, it dramatically decreases to a negligible amount.

Figure 5 shows the variation of $\mathrm{CV}$ values for five different energies and six standard irradiation geometries. The logarithmic scale of the Y-axis implies the remarkable variation of $\mathrm{CV}$ values by changing the source energy. It can be seen that for energies above $0.1 \mathrm{MeV}$, the $\mathrm{CV}$ value decreases to 
Table 1. The LADF mean values and their SDs (mean $\pm \mathrm{SD}$ ) in unit $\mathrm{pGy} \cdot \mathrm{cm}^{2}$ for broad beams of mono-energetic photons.

\begin{tabular}{|c|c|c|c|c|c|c|}
\hline PA & LLAT & RLAT & ROT & ISO & $\mathbf{A P}$ & $\begin{array}{l}\text { Energy } \\
(\mathrm{MeV})\end{array}$ \\
\hline $0.0009 \pm 0.0012$ & $0.00005 \pm 6 \mathrm{E}-5$ & $7.3 \mathrm{E}-6 \pm 4.8 \mathrm{E}-6$ & $0.0002 \pm 0.0003$ & $0.0002 \pm 0.0002$ & $0.0002 \pm 0.0005$ & 0.015 \\
\hline $0.0168 \pm 0.0062$ & $0.0015 \pm 0.0006$ & $0.001 \pm 0.0002$ & $0.0059 \pm 0.0023$ & $0.0045 \pm 0.0015$ & $0.0069 \pm 0.004$ & 0.02 \\
\hline $0.128 \pm 0.121$ & $0.0215 \pm 0.0024$ & $0.0205 \pm 0.0019$ & $0.0648 \pm 0.0057$ & $0.0518 \pm 0.0043$ & $0.079 \pm 0.011$ & 0.03 \\
\hline $0.218 \pm 0.010$ & $0.055 \pm 0.003$ & $0.0526 \pm 0.0023$ & $0.127 \pm 0.005$ & $0.105 \pm 0.004$ & $0.155 \pm 0.010$ & 0.04 \\
\hline $0.271 \pm 0.009$ & $0.083 \pm 0.003$ & $0.0798 \pm 0.0024$ & $0.170 \pm 0.003$ & $0.141 \pm 0.003$ & $0.204 \pm 0.008$ & 0.05 \\
\hline $0.309 \pm 0.008$ & $0.106 \pm 0.004$ & $0.101 \pm 0.002$ & $0.200 \pm 0.003$ & $0.168 \pm 0.002$ & $0.237 \pm 0.007$ & 0.06 \\
\hline $0.381 \pm 0.007$ & $0.142 \pm 0.004$ & $0.136 \pm 0.003$ & $0.256 \pm 0.002$ & $0.216 \pm 0.002$ & $0.297 \pm 0.007$ & 0.08 \\
\hline $0.461 \pm 0.008$ & $0.178 \pm 0.005$ & $0.171 \pm 0.003$ & $0.313 \pm 0.002$ & $0.266 \pm 0.002$ & $0.359 \pm 0.008$ & 0.1 \\
\hline $0.689 \pm 0.010$ & $0.280 \pm 0.007$ & $0.268 \pm 0.004$ & $0.475 \pm 0.003$ & $0.406 \pm 0.003$ & $0.539 \pm 0.011$ & 0.15 \\
\hline $0.933 \pm 0.014$ & $0.396 \pm 0.010$ & $0.381 \pm 0.006$ & $0.657 \pm 0.004$ & $0.561 \pm 0.004$ & $0.736 \pm 0.013$ & 0.2 \\
\hline $1.43 \pm 0.02$ & $0.657 \pm 0.016$ & $0.627 \pm 0.009$ & $1.04 \pm 0.01$ & $0.900 \pm 0.006$ & $1.15 \pm 0.02$ & 0.3 \\
\hline $1.92 \pm 0.03$ & $0.927 \pm 0.023$ & $0.890 \pm 0.013$ & $1.41 \pm 0.01$ & $1.24 \pm 0.01$ & $1.56 \pm 0.02$ & 0.4 \\
\hline $2.38 \pm 0.03$ & $1.21 \pm 0.03$ & $1.160 \pm 0.017$ & $1.79 \pm 0.01$ & $1.58 \pm 0.01$ & $1.97 \pm 0.03$ & 0.5 \\
\hline $2.81 \pm 0.03$ & $1.50 \pm 0.04$ & $1.44 \pm 0.02$ & $2.16 \pm 0.01$ & $1.92 \pm 0.01$ & $2.38 \pm 0.03$ & 0.6 \\
\hline $3.62 \pm 0.04$ & $2.07 \pm 0.05$ & $1.977 \pm 0.027$ & $2.87 \pm 0.02$ & $2.56 \pm 0.02$ & $3.12 \pm 0.04$ & 0.8 \\
\hline $4.38 \pm 0.04$ & $2.64 \pm 0.06$ & $2.52 \pm 0.03$ & $3.54 \pm 0.02$ & $3.18 \pm 0.02$ & $3.82 \pm 0.04$ & 1 \\
\hline $6.01 \pm 0.05$ & $3.96 \pm 0.08$ & $3.81 \pm 0.04$ & $5.04 \pm 0.03$ & $4.61 \pm 0.02$ & $5.39 \pm 0.05$ & 1.5 \\
\hline $7.45 \pm 0.06$ & $5.18 \pm 0.10$ & $5.00 \pm 0.05$ & $6.38 \pm 0.04$ & $5.90 \pm 0.02$ & $6.76 \pm 0.06$ & 2 \\
\hline $9.95 \pm 0.07$ & $7.38 \pm 0.14$ & $7.18 \pm 0.06$ & $8.81 \pm 0.1$ & $8.15 \pm 0.03$ & $9.19 \pm 0.06$ & 3 \\
\hline $12.2 \pm 0.1$ & $9.42 \pm 0.16$ & $9.19 \pm 0.07$ & $11.0 \pm 0.1$ & $10.3 \pm 0.05$ & $11.4 \pm 0.1$ & 4 \\
\hline $14.3 \pm 0.1$ & $11.4 \pm 0.2$ & $11.1 \pm 0.1$ & $13.0 \pm 0.1$ & $12.2 \pm 0.1$ & $13.4 \pm 0.1$ & 5 \\
\hline $16.3 \pm 0.1$ & $13.2 \pm 0.2$ & $12.9 \pm 0.1$ & $15.0 \pm 0.1$ & $14.1 \pm 0.1$ & $15.4 \pm 0.1$ & 6 \\
\hline $20.4 \pm 0.1$ & $16.9 \pm 0.3$ & $16.5 \pm 0.1$ & $18.8 \pm 0.1$ & $17.9 \pm 0.1$ & $19.3 \pm 0.1$ & 8 \\
\hline $24.3 \pm 0.2$ & $20.5 \pm 0.3$ & $20.1 \pm 0.1$ & $22.7 \pm 0.1$ & $21.6 \pm 0.1$ & $23.1 \pm 0.1$ & 10 \\
\hline
\end{tabular}

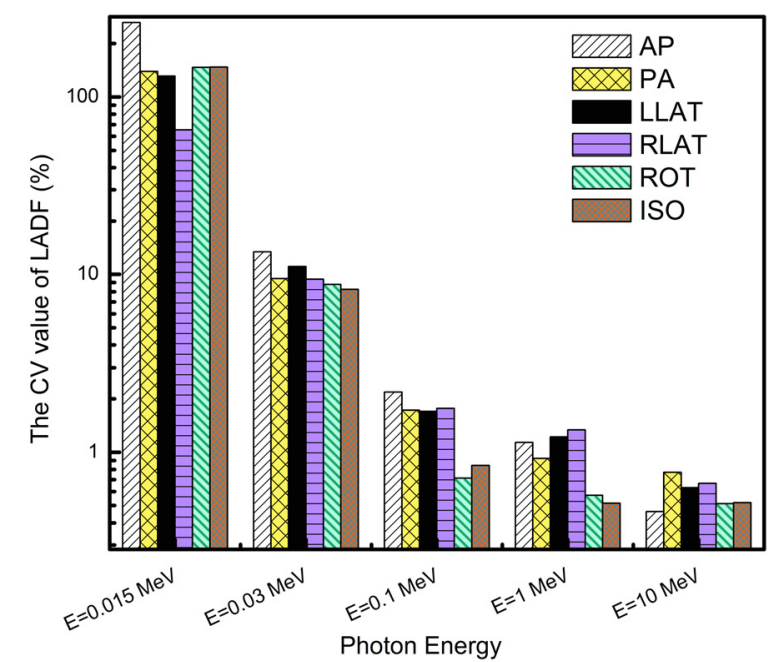

Fig. 5. Variation of the CV values in terms of photon energy in different irradiation geometries.

less than $2 \%$ for all exposure geometries. The range of fluctuation of the LADF for three lungs in terms of photon energy is also indicated in Figure 6. In this figure, the maximum (case 27), minimum (case 97) and average (case 89) amounts of the LADF and a cross-sectional plot of the related phantoms are shown for AP irradiation geometry.

\subsection{Mono-energetic neutron sources}

Table 2 summarizes the average values of LADFs and their SDs in the presence of mono-energetic neutron sources. Figures 7 and 8 provide additional information about the results which are given in Table 2. As shown in Figure 7, regardless of the irradiation geometry, the CV value of the LADFs is very small (less than $10 \%$ ). However, it has a peak at $1 \mathrm{MeV}$, which implies the higher fluctuation of the LADF in this energy.

The range of the LADF variation in terms of energy for PA geometry is illustrated in Figure 8. Similar to Figure 6, the data given in Figure 8 is related to the phantoms which have maximum (case 56), minimum (case 20) and average (case 73) LADFs.

\section{Discussion}

According to the data tabulated in Tables 1 and 2, for most irradiation conditions, the SD values of mean LADFs are very small and acceptable (less than $3 \%$ of the mean value), so that the dose received by the lung is approximately independent of its shape. Some exceptions are observed in situations in which phantoms were irradiated by low-energy photons or $1 \mathrm{MeV}$ neutrons.

The reason for high discrepancies in LADFs for lowenergy photon exposure is obvious. Because of the high probability of photoelectric interaction for low-energy photons and 
Table 2. The LADF mean values and their SDs (mean $\pm \mathrm{SD}$ ) in unit $\mathrm{pGy} \cdot \mathrm{cm}^{2}$ for broad beams of mono-energetic neutrons.

\begin{tabular}{ccccccc}
\hline Energy $(\mathbf{M e V})$ & AP & PA & LLAT & RLAT & ROT & ISO \\
\hline $\mathbf{2 . 5} \times \mathbf{1 0}^{-\mathbf{8}}$ & $0.897 \pm 0.022$ & $1.20 \pm 0.04$ & $0.406 \pm 0.004$ & $0.388 \pm 0.006$ & $0.768 \pm 0.01$ & $0.617 \pm 0.007$ \\
$\mathbf{1} \times \mathbf{1 0}^{-\mathbf{7}}$ & $1.18 \pm 0.03$ & $1.66 \pm 0.05$ & $0.525 \pm 0.006$ & $0.509 \pm 0.008$ & $1.02 \pm 0.01$ & $0.824 \pm 0.009$ \\
$\mathbf{1} \times \mathbf{1 0}^{-\mathbf{6}}$ & $1.74 \pm 0.05$ & $2.48 \pm 0.07$ & $0.759 \pm 0.017$ & $0.728 \pm 0.026$ & $1.50 \pm 0.02$ & $1.20 \pm 0.01$ \\
$\mathbf{1} \times \mathbf{1 0}^{-\mathbf{5}}$ & $2.02 \pm 0.05$ & $2.87 \pm 0.07$ & $0.873 \pm 0.011$ & $0.838 \pm 0.014$ & $1.74 \pm 0.02$ & $1.37 \pm 0.01$ \\
$\mathbf{1} \times \mathbf{1 0}^{-\mathbf{4}}$ & $2.09 \pm 0.05$ & $2.92 \pm 0.06$ & $0.906 \pm 0.012$ & $0.860 \pm 0.053$ & $1.79 \pm 0.01$ & $1.42 \pm 0.01$ \\
$\mathbf{1} \times \mathbf{1 0}^{-\mathbf{3}}$ & $2.12 \pm 0.05$ & $2.90 \pm 0.06$ & $0.908 \pm 0.012$ & $0.873 \pm 0.020$ & $1.80 \pm 0.01$ & $1.44 \pm 0.01$ \\
$\mathbf{1} \times \mathbf{1 0}^{-\mathbf{2}}$ & $2.12 \pm 0.14$ & $2.99 \pm 0.2$ & $0.911 \pm 0.012$ & $0.907 \pm 0.047$ & $1.78 \pm 0.01$ & $1.44 \pm 0.08$ \\
$\mathbf{0 . 0 2}$ & $2.16 \pm 0.05$ & $2.90 \pm 0.05$ & $0.916 \pm 0.012$ & $0.973 \pm 0.017$ & $1.81 \pm 0.01$ & $1.44 \pm 0.02$ \\
$\mathbf{0 . 0 5}$ & $2.23 \pm 0.05$ & $3.04 \pm 0.06$ & $0.951 \pm 0.014$ & $0.920 \pm 0.015$ & $1.89 \pm 0.01$ & $1.50 \pm 0.02$ \\
$\mathbf{0 . 1}$ & $2.38 \pm 0.05$ & $3.33 \pm 0.09$ & $1.01 \pm 0.015$ & $0.887 \pm 0.014$ & $2.02 \pm 0.02$ & $1.63 \pm 0.01$ \\
$\mathbf{0 . 2}$ & $2.69 \pm 0.09$ & $4.03 \pm 0.16$ & $1.14 \pm 0.02$ & $1.09 \pm 0.02$ & $2.34 \pm 0.05$ & $1.87 \pm 0.03$ \\
$\mathbf{0 . 5}$ & $4.00 \pm 0.21$ & $6.73 \pm 0.38$ & $1.62 \pm 0.05$ & $1.53 \pm 0.04$ & $3.64 \pm 0.14$ & $2.91 \pm 0.10$ \\
$\mathbf{1}$ & $5.30 \pm 0.35$ & $9.35 \pm 0.58$ & $2.11 \pm 0.08$ & $1.99 \pm 0.07$ & $4.98 \pm 0.22$ & $4.00 \pm 0.16$ \\
$\mathbf{2}$ & $13.7 \pm 0.5$ & $3.04 \pm 0.06$ & $6.00 \pm 0.16$ & $5.70 \pm 0.15$ & $12.4 \pm 0.3$ & $10.4 \pm 0.2$ \\
$\mathbf{3}$ & $20.0 \pm 0.6$ & $21.0 \pm 0.7$ & $9.70 \pm 0.22$ & $9.23 \pm 0.21$ & $18.1 \pm 0.3$ & $15.4 \pm 0.2$ \\
$\mathbf{5}$ & $29.7 \pm 0.6$ & $28.2 \pm 0.7$ & $16.2 \pm 0.3$ & $15.47 \pm 0.28$ & $26.9 \pm 0.3$ & $23.4 \pm 0.2$ \\
$\mathbf{1 0}$ & $44.9 \pm 0.7$ & $38.8 \pm 0.7$ & $27.7 \pm 0.4$ & $26.56 \pm 0.35$ & $41.1 \pm 0.3$ & $36.4 \pm 0.2$ \\
$\mathbf{1 4}$ & $54.0 \pm 0.7$ & $54.7 \pm 0.7$ & $34.9 \pm 0.4$ & $33.52 \pm 0.39$ & $49.7 \pm 0.3$ & $44.2 \pm 0.2$ \\
$\mathbf{2 0}$ & $64.0 \pm 0.6$ & $63.5 \pm 0.6$ & $44.2 \pm 0.5$ & $42.70 \pm 0.54$ & $59.2 \pm 0.4$ & $53.8 \pm 0.2$ \\
\hline
\end{tabular}
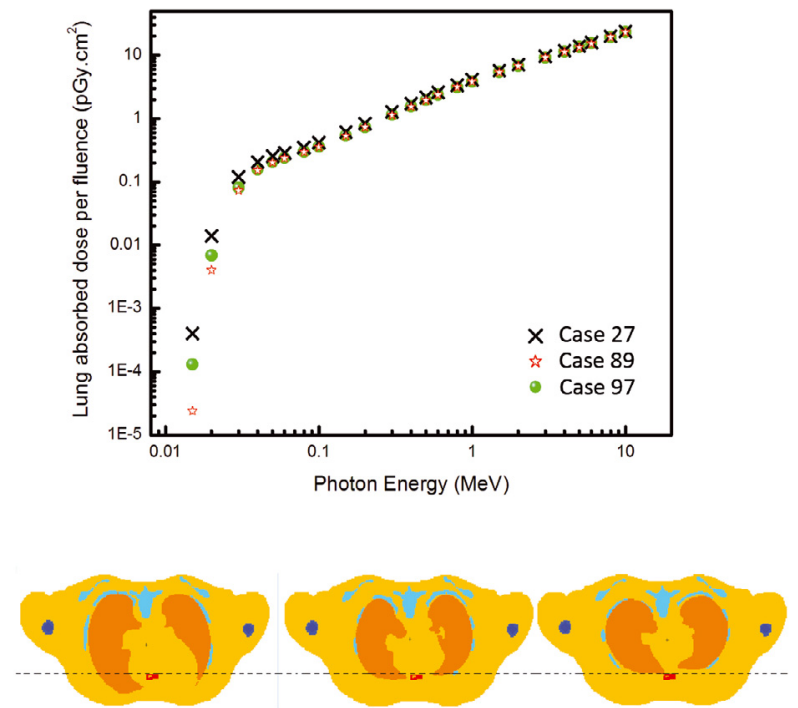

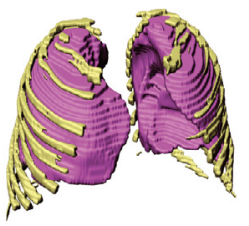

Case 27

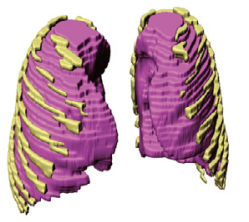

Case 89

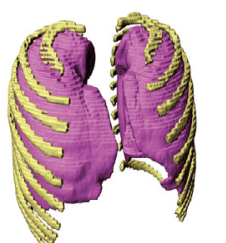

Case 97
Fig. 6. Variation of the LADF in terms of photon energy for three different phantoms in AP geometry.

their weak penetrating powers, the shape of the lung and the thickness of the tissue around the lung boundary would be significant parameters in the amount of dose delivered to the lung. Although the location of the lung was fixed in all constructed

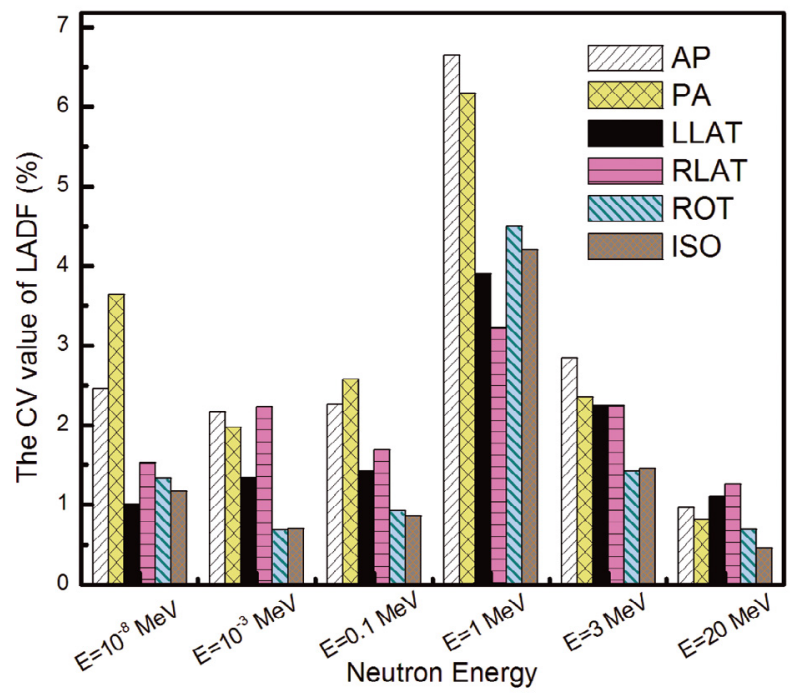

Fig. 7. Variation of the $\mathrm{CV}$ values in terms of neutron energy in different irradiation geometries.

phantoms, due to the different shapes of the lungs, various thicknesses of overlying tissues would cover this organ. Considering Figure 6, the lung of the phantom named case 89 has an average height, width and depth (Fig. 2). The lungs of case 27 and case 97 are somewhat different from case 89 . The depth and height of the lung of case 27 have higher and smaller values than those of case 89 , respectively. In contrast, the lung of case 97 has a greater height and smaller depth than that of case 89. In consequence, because of the similar volumes of the lungs, varieties in shape cause some differences in the positions of the lung boundaries with respect to the body surface. This discrepancy is due to the lesser shielding effect of 


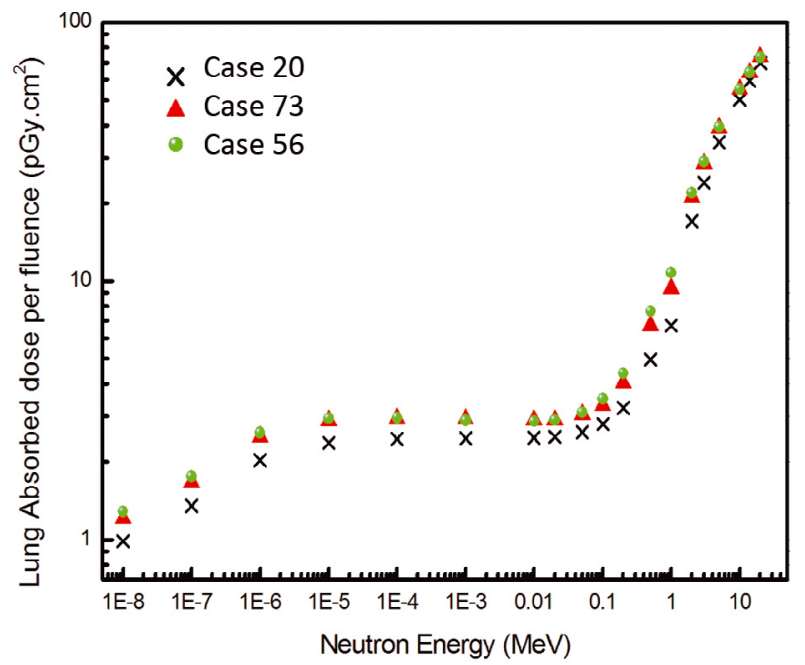

Fig. 8. Variation of the LADF in terms of neutron energy for three different phantoms in PA geometry.

the thinner muscle or residual tissue in case 89 and the greater shielding effect of the thicker surrounding layers in PAT097. Given the greater penetrating powers of high-energy photon beams, discrepancies decrease by increasing photon energy.

In the case of neutron sources, the $\mathrm{CV}$ values obtained of LADFs are small, generally less than $8 \%$, for all investigated situations (see Tab. 2 and Fig. 7). However, the LADFs of this set of statistical phantoms in all geometry irradiations show large differences at intermediate neutron energies $(1 \mathrm{MeV})$, while there is good agreement at other neutron energies. It is worth mentioning that for low-energy neutron irradiation, the photon-induced neutron plays an important role in the energy deposition process. On the other hand, the energy of the produced photons is sufficient for covering the lung volume; so the value of the LADF is approximately independent of the lung shape and position. For higher neutron energies, the secondary particles such as recoiled protons have a dominant role in the amount of deposited energy. Since a proton is a shortrange particle, the lung shape and the distance between the lung boundaries and body surface become significant parameters in the determination of the lung absorbed dose. This dependency is observed for energies up to $4 \mathrm{MeV}$, for which the energy transferred to the recoiled proton becomes larger. Under these conditions, a relatively uniform distribution of the energy deposition is provided for the lung and the $\mathrm{CV}$ value of LADFs decreases to less than $2 \%$.

A similar calculation was performed for a set of statistical phantoms in which the lung volume was considered as a statistical parameter. The behavior of the $\mathrm{CV}$ value and a detailed explanation about the energy deposition under different irradiation conditions can be found in the previous paper (Ebrahimi Khankook et al., 2014).

\section{Conclusion}

The present paper studied the effect of lung shape on LADF amounts from external photon and neutron exposure for six standard irradiation geometries. A population of phantoms with different lung shapes was needed for accurate investigation. Fixing other anatomical parameters, 70 lungs and their corresponding rib cages were extracted from medical images and imported into the AM reference ICRP phantom. The results obtained implied that if the volume and position of the imported lungs remain constant, the variation in the lung shape would not affect the lung absorbed dose significantly. Only in a few cases (low-energy photons up to $0.04 \mathrm{MeV}$ ) were the $\mathrm{CV}$ values of LADFs larger than $10 \%$. This means that the shape of the organ cannot be an essential parameter in the external dosimetry.

Acknowledgements. The authors gratefully acknowledge the important contributions of the heads of the medical imaging centers of the Imam Hossein Hospital of Tehran and Imam Reza Hospital of Mashhad. In addition, the authors would like to thank Mr. Piri, the manager of the Parsian Imaging Center of Mashhad, for his expert contribution for providing a large collection of radiography, CT and HRCT images.

\section{References}

Cassola V.F., Lima V.J.M, Kramer R., Khoury H.J. (2010) FASH and MASH: female and male adult human phantoms based on polygon mesh surfaces: I. Development of the anatomy, Phys. Med. Biol. 55, 133-162.

Ebrahimi Khankook A., Miri Hakimabad H., Rafat Motavalli L. (2014) The feasibility study of using lung statistical phantoms to determine the uncertainty of the lung absorbed dose in broad beams incident of photons and neutrons, Radiat. Prot. Dosim. (Revised).

Ferrari P., Gualdrini G. (2005) An improved MCNP version of the NORMAN voxel phantom for dosimetry studies, Phys. Med. Biol. 50, 4299-4316.

ICRP Publication 23 (1975) Report on the Task Group on Reference Man, pp. 1-480. Pergamon Press.

ICRP Publication 89 (2002) Basic Anatomical and Physiological Data for Use in Radiological Protection: Reference Values, pp. 1-265. Pergamon Press.

ICRP Publication 110 (2009) Adult Reference Computational Phantom, pp. 1-165. Elsevier Inc.

ICRP Publication 116 (2010) Conversion Coefficients for Radiological Protection Quantities for External Radiation Exposures, pp. 1-257. Elsevier Inc.

Karimi Shahri K., Rafat Motavalli L., Miri Hakimabad H. (2013) Can the same dose data be estimated from phantoms with different anatomies? Radioprotection 48, 527-544.

Kramer R., Zankl M., Williams G., Drexler G. (1982) The calculation of dose from external photon exposures using reference human phantoms and Monte Carlo methods: Part I. The male (ADAM) and female (EVA) adult mathematical phantoms GSF-Report S-885 (Neuherberg-Muenchen: Institut fuer Strahlenschutz, GSF-Forschungszentrum fuer Umwelt und Gesundheit) (reprint July 1999).

Kramer R., Vieira J.W., Khoury H.J., Lima F.R.A., Fuelle D. (2003) All about MAX: a male adult voxel phantom for Monte Carlo calculations in radiation protection dosimetry, Phys. Med. Biol. 48, 1239-1262. 
Kramer R., Khoury H.J., Vieira J.W., Loureiro E.C.M., Lima V.J.M., Lima F.R.A., Hoff G. (2004) All about FAX: a female adult voxel phantom for Monte Carlo calculation in radiation protection dosimetry, Phys. Med. Biol. 49, 5203-5216.

Kramer R., Khoury H.J., Vieira J.W., Lima V.J.M. (2006) MAX06 and FAX06: update of two adult human phantoms for radiation protection dosimetry, Phys. Med. Biol. 51, 3331-3346.

Kramer R., Cassola V.F., Khoury H.J., Vieira J.W., de Melo Lima V.J., Brown K.R. (2010) FASH and MASH: female and male adult human phantoms based on polygon mesh surfaces: II. Dosimetric calculations, Phys. Med. Biol. 55, 163-189.

Lee C., Lee J., Lee C. (2004) Korean adult male voxel model KORMAN segmented from magnetic resonance images, Med. Phys. 31, 1017-1022.

Lee C., Lee C., Park S.H., Lee J.K. (2006) Development of the two Korean adult tomographic computational phantoms for organ dosimetry, Med. Phys. 33, 380-390.

Li J., Qiu R., Zhang Z., Liu L., Zeng Z., Bi L., Li W. (2009) Organ dose conversion coefficients for external photon irradiation using the Chinese voxel phantom (CVP), Radiat. Prot. Dosim. 135, 33-42.

Sato K., Noguchi H., Emoto Y., Koga S., Saito K. (2007) Japanese adult male voxel phantom constructed on the basis of CT images, Radiat. Prot. Dosim. 123, 337-344.

Saito K., Wittmann A., Koga S., Ida Y., Kamei T., Funabiki J., Zankl M. (2001) Construction of a computed tomographic phantom for a Japanese male adult and dose calculation system, Radiat. Environ. Biophys. 40, 69-76.
Saito K., Sato K., Kinase S., Nagaoka T. (2009) Japanese Computational Phantoms: Otoko, Onago, JM, JM2, JF, TARO, HANAKO, Pregnant Woman, and Deformable Child. Handbook of anatomical models for radiation dosimetry (X.G. Xu and K.F. Eckerman, Eds.) pp. 221-253. CRC Press, Taylor \& Francis Group.

Xu X.G., Liu T. (2011). Quantifying uncertainty in radiation protection dosimetry using statistical phantoms. In: The Third International Workshop on Computational Phantoms for Radiation Protection, Imaging and Radiotherapy, August 8-9, 2011, Tsinghua University, Beijing, http://www.virtualphantoms. org/3rdWorkshopInBeijing.html).

Zankl M., Wittmann, A. (2001) The adult male voxel model "Golem" segmented from whole-body CT patient data, Radiat. Environ. Biophys. 40, 153-162.

Zhang B., Ma J., Liu L., Cheng J. (2007) CNMAN: a Chinese adult male voxel phantom constructed from color photographs of a visible anatomical data set, Radiat. Prot. Dosim. 124, 130-136.

Zhang B., Ma J., Zhang G., Liu Q., Qiu R., Li J. (2009a) Chinese Voxel Computational Phantoms: CNMAN, VCH, and CVP. In: Handbook of Anatomical Models for Radiation Dosimetry (X.G. XU and K.F. Eckerman, Eds.) pp. 279-303. CRC Press, Taylor \& Francis Group.

Zhang J., Na Y.H., Caracappa P.F., Xu X.G. (2009b) RPI-AM and RPI-AF, a pair of mesh-based, size-adjustable adult male and female computational phantoms using ICRP-89 parameters and their calculations for organ doses from monoenergetic photon beams, Phys. Med. Biol. 54, 5885-5908.

Cite this article as: A. Ebrahimi Khankook, H. Miri Hakimabad, L. Rafat Motavalli. A study of the effect of the lung shape on the lung absorbed dose in six standard photon and neutron exposure geometries. Radioprotection 50(1), 65-72 (2015). 